\title{
Spreading Depolarizations and Neurological Deterioration in Patients with Subdural Hematoma: Putting a Name to a Face?
}

\author{
David Robinson*
}

๑ 2022 Springer Science+Business Media, LLC, part of Springer Nature and Neurocritical Care Society

Chronic subdural hematomas (cSDHs) are an increasingly common disease encountered by neurointensivists [1]. Episodic neurologic symptoms have been well described in patients with $\operatorname{cSDH}[2,3]$, and although some syndromes are caused by focal seizures or other pathologies, many symptoms are unexplained [4]. These unexplained symptoms were recently classified as a new entity-nonepileptic, stereotypical, and intermittent symptoms (NESIS)-and were hypothesized to result from spreading depolarizations (SDs) [4]. SDs are pathologic waves in cerebral gray matter defined by the near-complete breakdown of electrochemical membrane gradients, with consequent silencing of electrical activity (spreading depression) [5]. The hypothesis that SDs underlie some of these syndromes is supported by the known role of SDs in delayed neurological injury [5], including in patients with cSDH [6].

In this issue of Neurocritical Care, Meadows et al. [7] expand on prior work by examining the clinical characteristics and significance of definite and "probable" SDs in postoperative patients with $\mathrm{cSDH}$. The authors combined two cohorts for this study: one cohort consisted of prospectively enrolled patients with cSDH with strip electrodes, whereas a second and larger cohort consisted of postoperative patients with cSDH who underwent usual care without a strip electrode. The prospective cohort was definitively evaluated for SDs using strip electrode data. In the cohort of patients without a strip electrode, the authors attempted to identify probable SDs

\footnotetext{
*Correspondence: Robin2dv@ucmail.uc.edu Department of Neurology and Rehabilitation Medicine, University of Cincinnati, Cincinnati, OH, USA
}

This article is part of the Collection Title on Spreading Cortical Depolarization

\section{Springer}

by looking for patients who were connected to electroencephalograms for changes at examination but who were not found to have epileptiform activity. They hypothesized that this cohort represented patients with NESISlike syndromes, and were thus likely to have SDs. In support of this hypothesis, the authors found that the rate of probable SDs in the retrospective cohort was similar to the rate of definite SDs in the prospective cohort $(22 \%$ vs. $15 \%$, respectively). Ultimately, they found that patients with probable SDs had worse functional outcome and a longer length of stay on multivariable analysis. These results raise the hope that SDs could be a therapeutic target in patients with cSDH.

Despite their increasing importance, cSDHs remain understudied [8], and the authors should be commended for their efforts to identify novel therapeutic targets in this disease. Nevertheless, there are some limitations to the conclusions that can be drawn from this study. Importantly, their method of identifying probable SDs has never been validated; they did not apply a rigorous definition of NESIS to the neurological syndromes, and a diverse number of pathologies (including seizures) could lead to neurologic deterioration with a negative electroencephalogram. Further, the study was limited to postoperative patients with $\mathrm{cSDH}$, even though nonoperative patients with cSDH also experience transient neurological syndromes.

Overall, the results of this study are an important step toward evaluating SDs as a therapeutic target in patients with cSDH. Still, more work is needed to definitively unite clinical manifestations with neurophysiological evidence of SDs. Better methods for the noninvasive detection of SDs would help immeasurably with this goal and would also identify the patients who are more likely to benefit from SD-targeted treatments in future trials. Until then, it will be difficult for most clinicians to feel 
confident in naming SDs as the cause of neurologic deterioration in their patients.

\section{Author contributions}

All authorship requirements have been met, and the author approves the final manuscript.

\section{Source of support}

This work received no funding.

\section{Conflicts of interest}

The author has no conflicts of interest to disclose.

\section{Publisher's Note}

Springer Nature remains neutral with regard to jurisdictional claims in published maps and institutional affiliations.

\section{Published online: 6 October 2021}

\section{References}

1. Balser D, Farooq S, Mehmood T, Reyes M, Samadani U. Actual and projected incidence rates for chronic subdural hematomas in United
States Veterans Administration and civilian populations. J Neurosurg. 2015;123(5):1209-15.

2. Kaminski HJ, Hlavin ML, Likavec MJ, Schmidley JW. Transient neurologic deficit caused by chronic subdural hematoma. Am J Med. 1992;92(6):698-700.

3. Moster ML, Johnston DE, Reinmuth OM. Chronic subdural hematoma with transient neurological deficits: a review of 15 cases. Ann Neurol. 1983;14(5):539-42.

4. Levesque M, lorio-Morin C, Bocti C, Vézina C, Deacon C. Nonepileptic, stereotypical, and intermittent symptoms (NESIS) in patients with subdural hematoma: proposal for a new clinical entity with therapeutic and prognostic implications. Neurosurgery. 2020;87(1):96-103.

5. Hartings JA, Shuttleworth CW, Kirov SA, Ayata C, Hinzman JM, Foreman $B$, et al. The continuum of spreading depolarizations in acute cortical lesion development: examining Leao's legacy. J Cereb Blood Flow Metab. 2017;37(5):1571-94.

6. Mohammad LM, Abbas M, Shuttleworth CW, Ahmadian R, Bhat A, Hill $\mathrm{DA}$, et al. Spreading depolarization may represent a novel mechanism for delayed fluctuating neurological deficit after chronic subdural hematoma evacuation. J Neurosurg. 2020;134(3):1294-302.

7. Meadows C, Davis H, Mohammad L, Shuttleworth CW, Torbey M, Zhu Y, et al. Spreading depolarization after chronic subdural hematoma evacuation: associated clinical risk factors and influence on clinical outcome. Neurocrit Care. 2021;1:1-9.

8. Frontera JA, Egorova N, Moskowitz AJ. National trend in prevalence, cost, and discharge disposition after subdural hematoma from 1998-2007. Crit Care Med. 2011;39(7):1619-25. 